\title{
A Tomographical Characterization of L-Convex Polyominoes
}

\author{
Giusi Castiglione ${ }^{1}$, Andrea Frosini ${ }^{2}$, Antonio Restivo ${ }^{1}$, and Simone Rinaldi ${ }^{2}$ \\ 1 Università di Palermo, Dipartimento di Matematica e Applicazioni, \\ via Archirafi, 34 - 90123 Palermo, Italy \\ \{giusi, restivo\}@math.unipa.it. \\ 2 Università di Siena, Dipartimento di Scienze Matematiche ed Informatiche, \\ Pian dei Mantellini, 44 - 53100 Siena, Italy \\ frosini, rinaldi\}@unisi.it.
}

\begin{abstract}
Our main purpose is to characterize the class of L-convex polyominoes introduced in [3] by means of their horizontal and vertical projections. The achieved results allow an answer to one of the most relevant questions in tomography i.e. the uniqueness of discrete sets, with respect to their horizontal and vertical projections. In this paper, by giving a characterization of L-convex polyominoes, we investigate the connection between uniqueness property and unimodality of vectors of horizontal and vertical projections. In the last section we consider the continuum environment; we extend the definition of L-convex set, and we obtain some results analogous to those for the discrete case.
\end{abstract}

\section{Definitions and Preliminaries}

Let our environment be the integer lattice $\mathbb{Z} \times \mathbb{Z}$. A discrete set is a finite subset $S$ of $\mathbb{Z} \times \mathbb{Z}$ considered up to translations.

Usually, a discrete set is represented by a binary matrix or by a set of cells (unitary squares), as depicted in Fig. 1. In the sequel, we will use the latter representation, and we number the rows and the columns of the set starting from the upper left corner of the minimum rectangle containing it. We denote by $(i, j)$ the cell in the $i$-th row and $j$-th column of the rectangle.

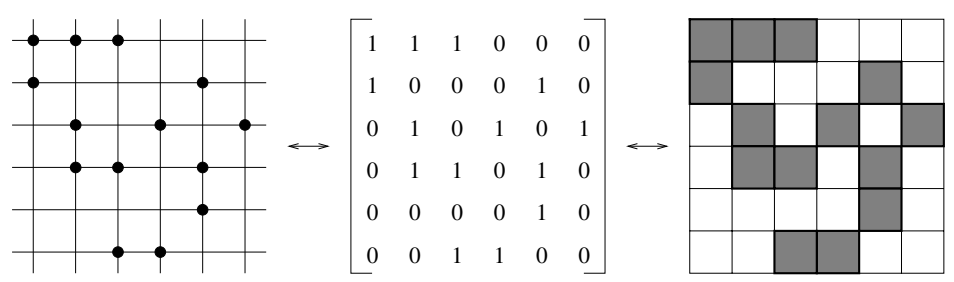

Fig. 1. A finite set of $\mathbb{Z}^{2}$, and its representation in terms of a binary matrix and a set of cells 

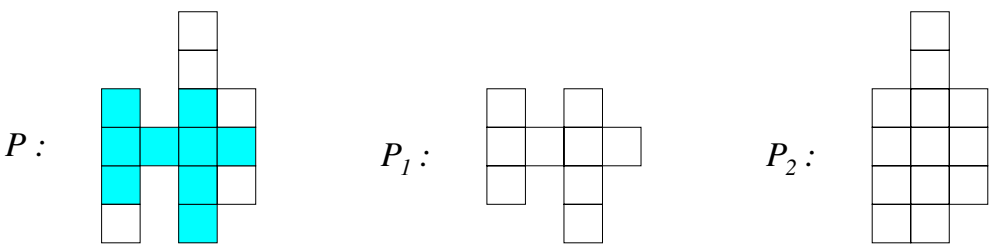

Fig. 2. The polyomino $P$ contains the polyomino $P_{1}$, but does not contain the polyomino $P_{2}$. The shaded cells of $P$ show the inclusion

In this paper we study a particular class of discrete sets, i.e. the well known class of polyominoes (cf. [4). A polyomino is defined as a finite union of cells whose interior is connected. Given two polyominoes $P$ and $P^{\prime}$, we say that $P$ is contained in $P^{\prime}$ if $P \subseteq P^{\prime}$ with respect to the standard set-inclusion (see Fig. (2).

A polyomino is said to be $h$-convex (resp. $v$-convex) if every its row (resp. column) is connected. A polyomino is said to be $h v$-convex, or simply convex, if it is both h-convex and v-convex (see Fig. 31).

For any two cells $A$ and $B$ in a polyomino, a path $\Pi_{A B}$, from $A$ to $B$, is a sequence $\left(i_{1}, j_{1}\right),\left(i_{2}, j_{2}\right), \ldots,\left(i_{r}, j_{r}\right)$ of adjacent disjoint cells, with $A=\left(i_{1}, j_{1}\right)$, and $B=\left(i_{r}, j_{r}\right)$. For each $1 \leq k<r$, we say that the two consecutive cells $\left(i_{k}, j_{k}\right),\left(i_{k+1}, j_{k+1}\right)$ form:

- an east step if $i_{k+1}=i_{k}+1$ and $j_{k+1}=j_{k}$;

- a north step if $i_{k+1}=h_{i}$ and $j_{k+1}=j_{k}+1$;

- a west step if $i_{k+1}=h_{i}-1$ and $j_{k+1}=j_{k}$;

- a south step if $i_{k+1}=h_{i}$ and $j_{k+1}=j_{k}-1$.

Finally, we define a path to be monotone if it is entirely made of only two of the four types of steps defined above.

The cells in a convex polyomino satisfy a particular connection property that involves the shape of the paths connecting any pair of them.

Proposition 1. A polyomino $P$ is convex iff every pair of cells is connected by a monotone path.

The property in Proposition 1 allows us to introduce a particular family of convex polyominoes, called $L$-convex polyominoes, defined and studied in 3 .

\subsection{The Class of L-Convex Polyominoes}

Let us consider a polyomino $P$. A path in $P$ has a change of direction in the cell $\left(i_{k}, j_{k}\right)$, for $2 \leq k \leq r-1$, if

$$
i_{k} \neq i_{k-1} \Longleftrightarrow j_{k+1} \neq j_{k} .
$$

In 3 it is proposed a classification of convex polyominoes based on the number of changes of direction in the paths connecting any two cells of a polyomino. 

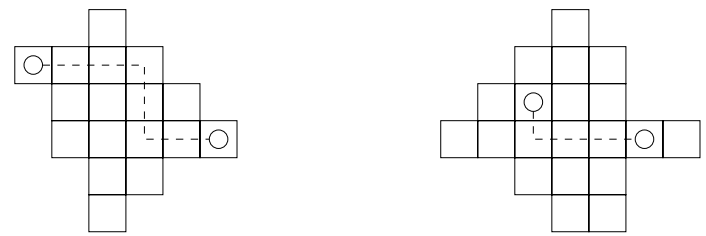

Fig. 3. The convex polyomino on the left is not L-convex, while the one on the right is L-convex. For both the polyominoes two cells are highlighted, and a monotone path which connects them and which contains the minimum number of possible changes of direction, is depicted

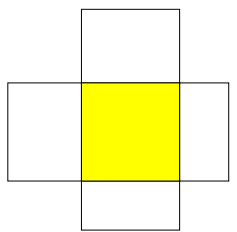

a)

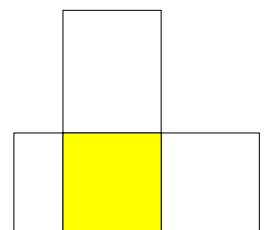

b)

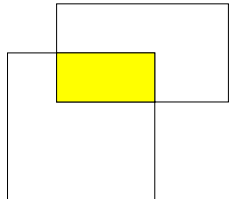

c)

Fig. 4. In $a$ ) and $b$ ) we have crossing intersections among the rectangles, while the intersection in $c$ ) is not crossing

More precisely, we call $k$-convex a convex polyomino such that every pair of its cells can be connected by a monotone path with at most $k$ changes of direction. For $k=1$ we have the class of $L$-convex polyominoes, i.e. those polyominoes such that every pair of their cells can be connected by a path with at most one change of direction (see Fig 3).

In the same paper it is given a nice characterization of L-convex polyominoes that involves the following notion of maximal rectangle.

A rectangle, that we denote by $[x, y]$, with $x, y \in \mathbb{N} \backslash\{0\}$, is a rectangular polyomino whose dimensions are $x$ and $y$ ( $x$ rows and $y$ columns). We say $[x, y]$ to be maximal in $P$ if

$$
\forall\left[x^{\prime}, y^{\prime}\right],[x, y] \subseteq\left[x^{\prime}, y^{\prime}\right] \subseteq P \Rightarrow[x, y]=\left[x^{\prime}, y^{\prime}\right]
$$

Two rectangles $[x, y]$ and $\left[x^{\prime}, y^{\prime}\right]$ contained in $P$ have a crossing intersection if their intersection is a rectangle having as basis the smallest of the two bases, and as height the smallest of the two heights, i.e.

$$
[x, y] \cap\left[x^{\prime}, y^{\prime}\right]=\left[\min \left\{x, x^{\prime}\right\}, \min \left\{y, y^{\prime}\right\}\right] \text {. }
$$

Figure 4 shows examples of crossing and non-crossing intersections.

Theorem 1. A convex polyomino is L-convex iff every pair of its maximal rectangles has crossing intersection. 

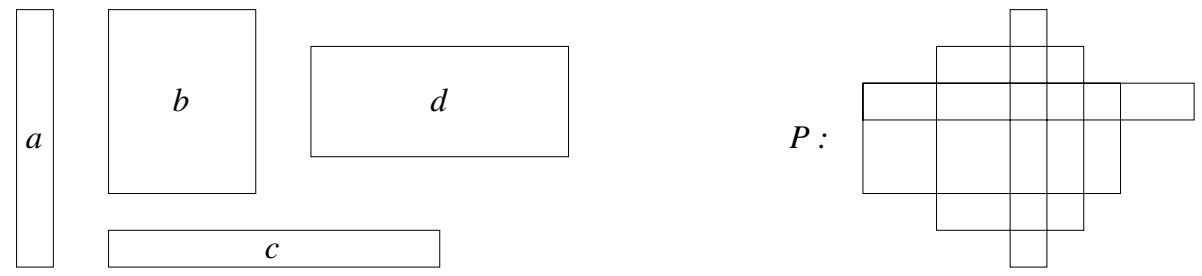

Fig. 5. A L-convex polyomino $P$ obtained by one of the overlappings of the four comparable rectangles $a, b, c$ and $d$ having crossing intersection

From Theorem 1, it immediately follows that all the maximal rectangles of a L-convex polyomino are distinct. The same result allows to characterize a L-convex polyomino as one of the overlapping of its maximal rectangles.

Since the set of maximal rectangles can be partially ordered as follows:

$$
\left[x_{1}, y_{1}\right]>\left[x_{2}, y_{2}\right] \text { if } x_{1}>x_{2} \text { and } y_{1}<y_{2},
$$

then each finite overlapping of comparable rectangles such that any pair of them has a crossing intersection, determines a L-convex polyomino (see Fig $[5$ for an example).

\subsection{Basic Notions of Discrete Tomography}

To each discrete set $S$, we can associate two integer vectors $H=\left(h_{1}, \ldots, h_{m}\right)$ and $V=\left(v_{1}, \ldots, v_{n}\right)$ such that, for each $1 \leq i \leq m, 1 \leq j \leq n, h_{i}$ and $v_{j}$ are the number of cells of $S$ which lie on row $i$ and column $j$, respectively. The vectors $H$ and $V$ are called the horizontal and vertical projections of $S$, respectively. Given two vectors $H$ and $V$, we will denote by $\mathcal{U}(H, V)$ the class of discrete sets having $H$ and $V$ as projections.

A discrete set $S$ is unique (with respect to $\mathrm{H}$ and $\mathrm{V}$ ) if $\mathcal{U}(H, V)=\{S\}$. In such a case also $H$ and $V$ are said to be unique.

Fundamental problems of discrete tomography concern the retrieval of information about some geometrical aspects (cf. 1, 2, 2]) of discrete sets, from the knowledge of their projections (for a survey cf. [5]).

In general, the horizontal and vertical projections of a discrete set are not sufficient to uniquely determine it (see Fig. 7), as it is known from [9], where Ryser pointed out that a discrete set is unique if and only if it does not contain particular configurations of points called switching components. Figure 6 shows the two simplest of them, called elementary switching components, and defined as follows: a discrete set $S$ contains the elementary switching component $a$ ) [resp. $b)]$ if there exists two rows $i$ and $i^{\prime}$, and two columns $j$ and $j^{\prime}$ such that the cells in positions $(i, j)$, and $\left(i^{\prime}, j^{\prime}\right)$ [resp. $\left(i^{\prime}, j\right)$, and $\left.\left(i, j^{\prime}\right)\right]$ belong to $S$ (represented in the figure by filled squares), while the cells in positions $\left(i^{\prime}, j\right)$, and $\left(i, j^{\prime}\right)$ [resp. $(i, j)$, and $\left.\left(i^{\prime}, j^{\prime}\right)\right]$ do not belong to $S$ (represented in the figure by dotted squares). 


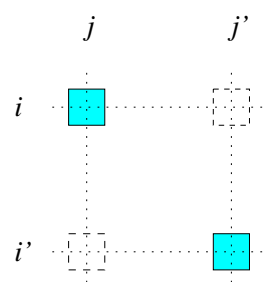

a)

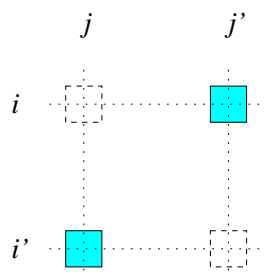

b)

Fig. 6. The two elementary switching components. The presence of one of them in a discrete set assures its non-uniqueness
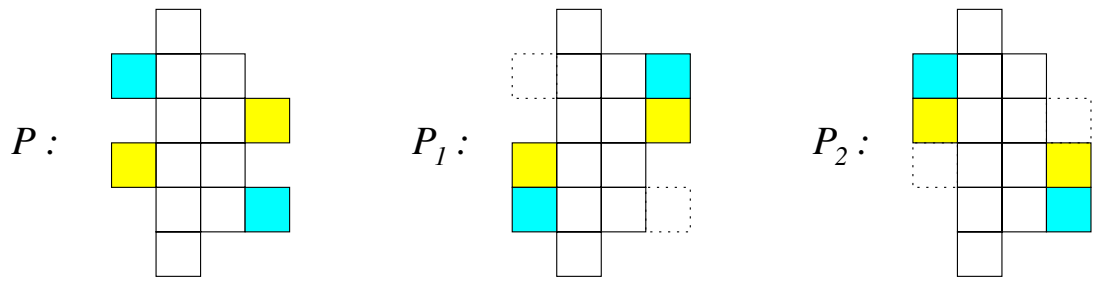

Fig. 7. Three polyominoes belonging to the class $\mathcal{U}(H, V)$, with $H=(1,3,3,3,3,1)$ and $V=(2,6,4,2)$

Furthermore, Ryser defined an operator, called interchange and successively switching (operator), which modifies a discrete set by changing one of its switching component, if it exists, into the other.

In Fig. 7 the discrete sets $P_{1}$ and $P_{2}$ are obtained from $P$ by performing the two highlighted switchings.

Clearly, switching does not modify the projections of a discrete set, which consequently reveals to be non-unique (cf. [10]). The reverse of this property is also true, as stated in the following

Theorem 2. (Ryser's Theorem) A discrete set is non-unique (with respect to its horizontal and vertical projections) if and only if it has a switching component.

\section{A Characterization Theorem for L-Convex Polyominoes}

In this section we furnish a series of results that produce a characterization of L-convex polyominoes in terms of horizontal and vertical projections.

Lemma 1. A L-convex polyomino $P$ is uniquely determined by its horizontal and vertical projections. 
Proof. Theorem 2 allows us to achieve the uniqueness of $P$ by proving that it does not contain any switching component. So, let us assume that there exists a switching component involving the two cells $A$ and $B$ of $P$, in positions $(i, j)$ and $\left(i^{\prime}, j^{\prime}\right)$, respectively, where $i \neq i^{\prime}$ and $j \neq j^{\prime}$. By definition of switching, the two cells in positions $\left(i, j^{\prime}\right)$ and $\left(i^{\prime}, j\right)$ do not belong to $P$, and consequently a monotone path $\Pi_{A B}$ having at most one change of direction does not exist. This fact contradicts the hypothesis of L-convexity of $P$.

Lemma 2. Let $j$ and $j^{\prime}$ be two different columns of a L-convex polyomino $P$, such that $v_{j} \leq v_{j^{\prime}}$. For each row $i$ of $P$, if $(i, j) \in P$, then $\left(i, j^{\prime}\right) \in P$.

Proof. Let us proceed by contradiction and assume that there exists a row $i^{\prime}$ of $P$ such that $\left(i^{\prime}, j\right) \in P$ and $\left(i^{\prime}, j^{\prime}\right) \notin P$. Since $v_{j} \leq v_{j^{\prime}}$, there exists a row $i^{\prime \prime}$ such that $\left(i^{\prime \prime}, j\right) \notin P$ and $\left(i^{\prime \prime}, j^{\prime}\right) \in P$. These four cells form a switching component, a contradiction by Lemma 1

Obviously a result similar to that of Lemma 2 holds if $v_{j}>v_{j^{\prime}}$, and, furthermore, if we replace the two different columns of $P$ with two of its rows.

We define an integer vector $X=\left(x_{1}, \ldots, x_{k}\right)$ to be unimodal, if there exists $0 \leq i \leq k$, such that $x_{1} \leq x_{2} \leq \ldots, \leq x_{i}$ and $x_{i} \geq x_{i+1} \geq \cdots \geq x_{k}$.

Lemma 3. If $P$ is a L-convex polyomino then its horizontal and vertical projections are unimodal.

Proof. Let $P$ be a L-convex polyomino belonging to $\mathcal{U}(H, V)$, with $H \in \mathbb{N}^{m}$ and $V \in \mathbb{N}^{n}$. By Theorem 1, it follows that each element $h_{i}$ of $H$ is the basis of a maximal rectangle of $P$. Let us proceed by contradiction and assume $H$ to be non-unimodal, i.e. there exist $1 \leq i<j<k \leq m$ such that $h_{j}<h_{k}$ and $h_{j}<h_{i}$. The following three cases arise:

$h_{i}=h_{k}$ : the cells of $P$ lying on row $i$ and row $k$ belong to the same maximal rectangle, so $h_{j} \geq h_{i}$, a contradiction;

$h_{i}<h_{k}$ : the two values $h_{i}$ and $h_{j}$ are the bases of two different maximal rectangles. Since each pair of maximal rectangles has crossing intersection, then $h_{j} \geq h_{i}$, a contradiction;

$h_{i}>h_{k}$ : analogous to the previous case.

Since each element $v_{j}$ of $V$, with $j=1, \ldots, m$, is the height of a maximal rectangle of $P$, a similar reasoning leads to prove that also $V$ is unimodal.

The properties stated in Lemmas 2 and 3 directly follow from the definition of L-convexity. A less intuitive result is the characterization of L-convex polyominoes by means of the uniqueness and monotonicity of its projections.

Theorem 3. Let $P \in \mathcal{U}(H, V)$, with $H \in \mathbb{N}^{m}$ and $V \in \mathbb{N}^{n}$.

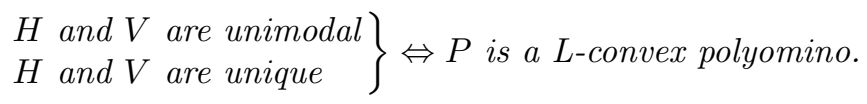


Proof. $(\Rightarrow)$ We prove by contradiction the $h$-convexity of $P$ : let us assume that there exist three cells $(i, j) \in P,\left(i, j^{\prime}\right) \notin P$ and $\left(i, j^{\prime \prime}\right) \in P$, with $i<i^{\prime}<i^{\prime \prime}$. The unimodality of $V$ allows the following three cases:

$v_{j} \geq v_{j^{\prime}} \geq v_{j^{\prime \prime}}$ : Lemma 2 applied to columns $j^{\prime \prime}$ and $j^{\prime}$, implies that $\left(i, j^{\prime}\right) \in$ $P$, which is clearly a contradiction;

$v_{j} \leq v_{j^{\prime}} \leq v_{j^{\prime \prime}}$ : Lemma2 2 applied to columns $j$ and $j^{\prime}$, implies that $\left(i, j^{\prime}\right) \in P$, a contradiction;

$v_{j} \leq v_{j^{\prime}}$ and $v_{j^{\prime}} \geq v_{j^{\prime \prime}}$ : Lemma 2 applied or to columns $j$ and $j^{\prime}$, or to columns $j^{\prime \prime}$ and $j^{\prime}$ implies that $\left(i, j^{\prime}\right) \in P$, again a contradiction.

A similar reasoning leads to the $v$-convexity of $P$.

Finally, for any pair of cells $(i, j)$ and $\left(i^{\prime}, j^{\prime}\right)$ belonging to $P$, the uniqueness of $P$ implies that $\left(i^{\prime}, j\right) \in P$ or $\left(i, j^{\prime}\right) \in P$, so the cells $(i, j)$ and $\left(i^{\prime}, j^{\prime}\right)$ can be connected by a path having at most one change of direction. This determines the connectedness and the L-convexity of $P$.

$(\Leftarrow)$ The result follows from Lemmas 1 and 3 .

The following remark is a direct consequence of the proof of Theorem 3

Remark 1. A convex discrete set is unique if and only if it is L-convex.

\section{Extension to Measurable Plane Sets}

In this section, we introduce the concept of L-convex plane set in order to extend to the continuum the uniqueness results stated in Section 2

In the case of generic measurable plane sets, G.G.Lorentz gave in [8] necessary and sufficient conditions for a pair of projections to be respectively unique, nonunique and consistent. These results were obtained by using analytic transformations of the projection functions. Further studies considered the same problem from a geometrical point of view, with the aim of defining a switching theory which translates in the continuum what was introduced for discrete sets. In particular in [6], the authors introduced the notion of switching components in the continuum, and stated a result similar to Theorem 2, Furthermore, they furnished other nice characterizations of plane sets related to their geometrical properties. In this section we will often rely on these works in order to support our results.

So, let us start by recalling the following standard definitions: a set $S$ of $\mathbb{R}^{2}$ is called $h$-convex (resp. $v$-convex) if, for each pair of points $(x, y),(u, v) \in S$, with $y=v$ (resp. $x=u$ ), the horizontal (resp. vertical) line segment which join them is entirely contained in $S$. We call $h v$-convex the plane sets that are both $h$-convex and $v$-convex.

Furthermore, a step polygon is a polygonal curve consisting of horizontal and vertical line segments and having no self-intersections. A step polygon joining two distinct points $(x, y),(u, v) \in \mathbb{R}^{2}$ can be represented as a finite sequence of vertices $\left(x_{0}, y_{0}\right),\left(x_{1}, y_{1}\right), \ldots,\left(x_{k}, y_{k}\right)$ such that each vertex is connected by a line segment to the next one, $\left(x_{0}, y_{0}\right)=(x, y)$, and $\left(x_{k}, y_{k}\right)=(u, v)$. To our purpose, line segments are the continuum counterpart of the four kinds of steps defined 


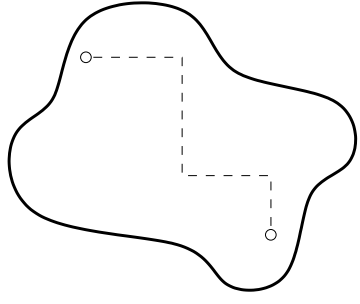

a)

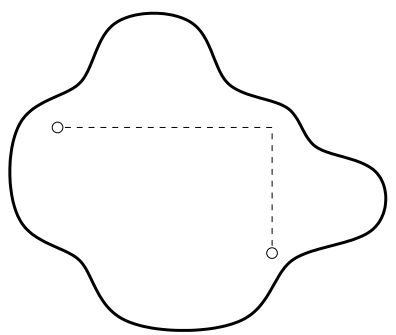

b)

Fig. 8. a) a hv-convex plane set and a monotone step polygon lying inside it; b) a Lconvex plane set, and two of its cells joined with a three vertex monotone step polygon

in Section 1 for the discrete lattice, and so they can be classified as north, south, east and west segments. A step polygon is called monotone if it is composed of at most two different kinds of these segments.

Hence, we have the following natural translation of Proposition 1 to $h v$-convex plane sets:

Proposition 2. A plane set $S$ is hv-convex iff every pair of points in $S$ can be joined by a monotone step polygon lying in $S$.

Now we can finally define a plane set $S$ to be $L$-convex if each pair of its points can be joined by a monotone step polygon with at most three vertices, and entirely contained in $S$ (see Fig 8 ).

\subsection{A Characterization Theorem for L-Convex Plane Sets}

In this paragraph, the reader will encounter some basic definitions together with the continuum counterparts of the results stated in Section 2

A function $f(x)$, defined in the interval $[a, b] \subset \mathbb{R}$, is unimodal if there exists $\bar{x} \in[a, b]$ (called mode) such that $f(x)$ increases from $a$ to $\bar{x}$ and decreases from $\bar{x}$ to $b$.

Let $S \subseteq \mathbb{R}^{2}$ be a measurable set such that $\lambda_{2}(S)<\infty\left(\lambda_{2}\right.$ being the two dimensional Lebesgue measure), and let $f(x, y)$ be its characteristic function. Using notations and definitions from 6, we call horizontal projection of $S$ the function

$$
f_{x}(y)=\int_{-\infty}^{\infty} f(x, y) d x
$$

and vertical projection of $S$ the function

$$
f_{y}(x)=\int_{-\infty}^{\infty} f(x, y) d y
$$


These functions exist almost everywhere on $\mathbb{R}$ and they are integrable (Fubini's theorem).

In [6], it is introduced a notion of switching components in the continuum which naturally extends the one for discrete sets.

In particular, let $t, u$ be two real numbers. The sets

$$
\begin{aligned}
S(t, 0) & =\{(x, y) \mid(x-t, y) \in S\} \\
S(0, u) & =\{(x, y) \mid(x, y-u) \in S\}
\end{aligned}
$$

are called horizontal and vertical translation of $S$, respectively.

We say that $S$ admits a switching component if there exist four sets $A, B, C, D$ and two real numbers $t$ and $u$ such that $B \cup C \subseteq S, A \cup D \cap S=\emptyset$, and such that $B=A_{(t, 0)}, C=A_{(0, u)}$ and $D=A_{(t, u)}$.

We have that if $S$ admits a switching component, then $S$ is not uniquely determined by its projections, in fact the set

$$
S^{\prime}=(S-(B \cup C)) \cup(A \cup D)
$$

is different from $S$, and it has its same horizontal and vertical projections.

The existence of a switching component is also a necessary condition to guarantee the non-uniqueness of the set $S$ (see [6] for a proof), so we have the following result, analogous to Lemma 1

Theorem 4. A measurable plane set having finite measure is non-uniquely determined by its projections iff it has a switching component.

Finally, L-convexity of a plane set causes the existence of a mode both in its horizontal and in its vertical projections:

Lemma 4. If a plane set is L-convex, then both its horizontal and its vertical projections are unimodal.

The proof can be simple inferred from that of Lemma 3. As a consequence we can obtain, for the continuous case, the same characterization result as for discrete sets:

Theorem 5. Let $f_{x}$ and $f_{y}$ be projection functions defined in $\mathbb{R}^{2}$ of a plane set S. It holds that

$$
\left.\begin{array}{l}
f_{x} \text { and } f_{y} \text { are unimodal } \\
f_{x} \text { and } f_{y} \text { are unique }
\end{array}\right\} \Leftrightarrow S \text { is L-convex. }
$$

A last remark is needed: in [6], a different and interesting characterization of unique plane sets is provided. Let $S$ be a measurable plane set of finite measure. The rectangle $X \times Y$ is measurably inscribed (briefly $m$-inscribed) in $S$ if

$$
X \times Y \subseteq S \quad \text { and } \quad \bar{X} \times \bar{Y} \subseteq \bar{S}
$$




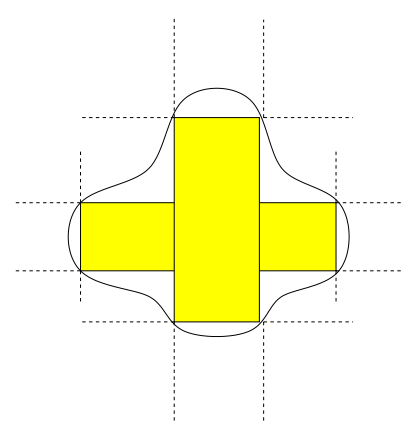

Fig. 9. Two $m$-inscribed rectangles inside a L-convex plane set

The set $S$ is $m$-inscribable if it is the union of $m$-inscribed rectangles. We can immediately argue that the presence of $m$-inscribed rectangles inside the set $S$ is similar to the presence of maximal rectangles inside an L-convex polyomino.

This idea is strengthened by the fact that, using Theorem 4, in [6] it is proved the following

Theorem 6. A measurable plane set having finite measure is uniquely determined by its projection functions iff it is $m$-inscribable.

We want to observe that the notion of crossing intersection in the continuum environment, leads to the equivalence between L-convex plane sets and $m$-inscribable plane sets. In fact, at the same time, we obtain a nice generalization of Theorem 1 and an uniqueness result.

Theorem 7. Let $S$ be a measurable plane set. It holds that $S$ is L-convex iff $S$ is $m$-inscribable by rectangles with crossing intersection.

\section{Conclusions and Further Work}

In this work we have proposed a characterization of L-convex sets in terms of features relevant to discrete tomography. In particular, we observed that each L-convex set is unique with respect to its horizontal and vertical projections, and that both the projections show a unimodal behavior. The characterization is achieved after showing that these two properties are also sufficient to obtain a L-convex set.

Finally, the last section of the paper concerns the natural extension of our main result to the continuum environment.

We would like to point out some open questions: one can ask wether similar tomographical characterizations can be proved when generalizing the notion of L-convexity by taking into consideration two or more directions different from (or possibly strictly including) the horizontal and the vertical ones. 
Furthermore, we could consider the extension of the notion of L-convex polyomino to the three dimensional lattice. In fact, it seems not so trivial to keep maintaining the crucial equivalence between the characterizations of L-convexity by means of monotone path and of maximal rectangles in such environment.

\section{References}

1. Barcucci, E., Del Lungo, A., Nivat, M., Pinzani, R.: Reconstructing convex polyominoes from horizontal and vertical projections. Theoret. Comput. Sci. 155 (1996) 321-347

2. Brualdi, R.A.: Matrices of zeros and ones with fixed row and column sum vectors. Lin. Algebra and Its Applications 33, (1980) 159-231

3. Castiglione, G., Restivo, A.: Reconstruction of L-convex Polyominoes. Electron. Notes in Discrete Math. 12 Elsevier Science (2003)

4. Golomb, S.W.: Polyominoes. Scribner, New York (1965)

5. Herman, G.T., Kuba, A. (eds.): Discrete Tomography: Foundations, Algorithms and Applications, Birkhauser Boston, Cambridge, MA (1999)

6. Kuba, A., Volčič, A.: Characterization of measurable plane sets which are reconstructable from their two projections. Inverse Problems 4 (1988) 513-527

7. Kuba, A., Balogh, E.: Reconstruction of convex 2D discrete sets in polynomial time Theoret. Comput. Sci. 283 (2002) 223-242

8. Lorentz, G.G.: A problem of plane measure. Am. J. Math. 71 (1949) 417-426

9. Ryser, H.J.: Combinatorial properties of matrices of zeros and ones. Canad. J. Math. 9 (1957) 371-377

10. Shliferstein, A.R., Chien, Y.T.: Switching components and the ambiguity problem in the reconstruction of pictures from their projections. Pattern Recognition, 10 (1978) 327-340 